\title{
Chronic aerobic exercise improves insulin sensitivity

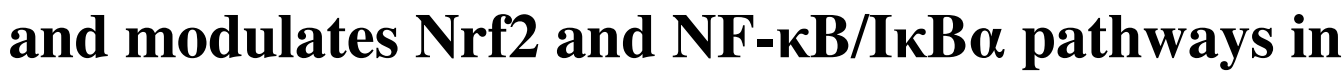 the skeletal muscle of rats fed with a high fat diet
}

\author{
QIAN YU, ZHENGYUN XIA, EMILY CHIU LIONG and GEORGE LIM TIPOE
}

\author{
School of Biomedical Sciences, Li Ka Shing Faculty of Medicine, The University of Hong Kong, \\ Hong Kong, SAR, P.R. China
}

Received March 19, 2019; Accepted August 12, 2019

DOI: $10.3892 / \mathrm{mmr} .2019 .10787$

\begin{abstract}
The present study aimed to investigate the molecular mechanisms of the ameliorative effects of chronic aerobic exercise on non-alcoholic steatohepatitis (NASH) in rat skeletal muscle. Female Sprague-Dawley rats ( $\mathrm{n}=6-9$ per group) were divided into four groups: i) Rats fed with normal chow; ii) exercise rats fed with normal chow + exercise (run on a rotarod for 30 min per day from 9-12 weeks); iii) rats fed with a high-fat diet (HFD); iv) rats fed with an HFD + exercise. All HFD rats were fed with an HFD consisting of $30 \%$ fat from fish oil throughout the study for 12 weeks. Exercise decreased the levels of hepatic lipogenic markers carbohydrate-responsive element-binding protein, fat-specific protein 27 and liver X receptor and improved systemic glucose and insulin intolerance in the NASH animal model. The beneficial effects may have been mediated partly via the tripartite motif-containing family protein 72 (TRIM72)/PI3K/Akt/mTOR pathway, accompanied with an upregulation of glucose transporter 4 in the skeletal muscle. The exercise regimen activated the master regulator of antioxidant enzymes, nuclear factor erythroid 2-related factor 2, with upregulation of superoxide dismutase $[\mathrm{Cu}-\mathrm{Zn}]$ expression and a corresponding decrease in kelch-like ECH-associated protein 1 expression, but failed to decrease the levels of the oxidative marker malondialdehyde in the HFD rat skeletal muscle. Chronic exercise decreased the expression of the inflammation marker NF- $\mathrm{kB}$, followed by a decrease in interleukin- 6 and tumor necrosis factor- $\alpha$ levels, as verified by a corresponding increase in the level of NF- $\mathrm{\kappa B}$ inhibitor $\alpha$ expression. Exercise may exert its beneficial effects by improving muscle insulin sensitivity via the TRIM72/PI3K/Akt/mTOR
\end{abstract}

Correspondence to: Dr George Lim Tipoe, School of Biomedical Sciences, Li Ka Shing Faculty of Medicine, The University of Hong Kong, 21 Sassoon Road, Pokfulam, Hong Kong, SAR, P.R. China

E-mail: tgeorge@hku.hk

Key words: fatty liver, skeletal muscle, inflammation, glucose tolerance, oxidative stress, hepatic lipogenesis pathway, contributing to the improvement of systemic insulin intolerance, and finally leading to decreased hepatic lipogenesis during NASH. The attenuation of insulin resistance by exercise may be partly achieved through a decrease in the level of inflammation and an increased antioxidant response.

\section{Introduction}

Non-alcoholic fatty liver disease (NAFLD) is one of the metabolic diseases affecting 20-30\% of the population in Western countries, which is characterised by an excess accumulation of fat in hepatocytes (1). In 10-25\% of subjects, NAFLD may progress to non-alcoholic steatohepatitis (NASH), which is characterised by histological features of hepatocyte ballooning and lobular inflammation $(2,3)$. As skeletal muscle accounts for $>80 \%$ of insulin-stimulated glucose from the blood stream and stored in the muscle as glycogen, it is a key tissue type contributing to the development of systemic insulin resistance (4). In recent years, skeletal muscle insulin resistance has been posited to contribute to the development of NAFLD by driving hepatic de novo lipogenesis of the ingested carbohydrates, as compared with skeletal muscle glycogen synthesis, leading to hypertriglyceridemia and increased hepatic triglyceride synthesis $(5,6)$. The hypothesis is further supported by data from experimental and epidemiological studies, suggesting the importance of skeletal muscle insulin resistance as a potential and promising therapeutic target for treating $\operatorname{NAFLD}(7,8)$.

At present, the precise molecular mechanisms of skeletal muscle insulin resistance remain unclear. Nevertheless, emerging evidence has demonstrated the roles of inflammation and oxidative stress in predisposition to insulin resistance: It has been indicated that hyperlipidaemia results in the increases in fatty acids uptake and production of fatty acids metabolites in the skeletal muscle, which promotes the inflammatory responses (9), and the pro-inflammatory cytokines generated in the skeletal muscle lead to insulin resistance by inhibiting insulin signal transduction with increased macrophage infiltration (10). Oxidative stress is the by-product of insufficient clearance of the cellular production of oxidants, including reactive oxygen species (ROS) and reactive nitrogen species (RNS), by the antioxidant defence system within the cell (11). Emerging data indicate that oxidative stress due to 
increased ROS and RNS generation and/or compromised antioxidant systems may serve a fundamental role in the aetiology of skeletal muscle insulin resistance $(12,13)$. Therefore, improving the understanding of the processes by which inflammation, oxidative stress and insulin resistance develop and interact in the pathogenesis of NAFLD may provide important breakthroughs for the prevention of and interventions for this disease.

Chronic aerobic exercise is considered as an effective treatment strategy for NAFLD. While a number of studies emphasise the benefits of exercise in the liver (14-16), few studies have considered the role of skeletal muscle in the amelioration of fatty liver as a result of chronic exercise. Skeletal muscle insulin resistance has recently been proposed to be a key factor in the progression of NAFLD; therefore, it is reasonable to assume that the therapeutic effect of exercise training on NAFLD may be partly dependent on the improvement of insulin sensitivity with an decreased inflammatory response and levels of oxidative stress in the skeletal muscle (5).

Of the multiple previously described NASH animal models $(17,18)$, the model described in the present study, which did not require a very high level of fat in the diet $30 \%$ fish oil), is more relevant and closer to the clinical condition (19). Although at low dosage ( $\leq 10 \%$ of total kcal), fish oil rich in $\omega-3$ polyunsaturated fatty acids has been widely demonstrated to be beneficial for NAFLD, for example improving hepatic lipid metabolism and regulating bile components $(20,21), 30 \%$ fish oil has been observed to cause dysfunctional lipid metabolism, particularly in female rats that are more susceptible to liver damage, according to our previous data (19). The present study investigated the molecular effects of a 4-week aerobic exercise regimen in the rat skeletal muscle, involving insulin resistance, inflammation and oxidative stress in the pathogenesis of NASH.

\section{Materials and methods}

Animal protocol. A total of 24-36 Female Sprague-Dawley rats (8-10 weeks old; $180-200 \mathrm{~g}$; $\mathrm{n}=6-9$ per group) were obtained from the Laboratory Animal Unit of The University of Hong Kong. The experimental groups maintained were in a controlled environment $\left(21^{\circ} \mathrm{C} ; 12: 12\right.$ light: Dark cycle) and divided into four groups: i) Rats fed with normal chow (PicoLabH Rodent Diet 20; LabDiet); ii) exercise rats fed with normal chow; iii) rats fed with a high-fat diet (HFD); iv) rats fed an HFD + exercise. All HFD rats were fed with $30 \%$ fat from fish oil (Sigma-Aldrich; Merck KGaA) throughout the study for 12 weeks, while the calories of normal chow contain $13 \%$ calories from fat. The preparation of HFD and the development of NASH animal model were performed as described previously (19). Rats in the exercise group were trained to run on a rotarod from 9-12 weeks (30 $\mathrm{min} /$ day, 6 days/week). The exercise protocol consisted of $5 \mathrm{~m} / \mathrm{min}$ for $3 \mathrm{~min}, 10 \mathrm{~m} / \mathrm{min}$ for $3 \mathrm{~min}$, and $20 \mathrm{~m} / \mathrm{min}$ for $24 \mathrm{~min}$. After the 12 th week, rats underwent terminal anaesthesia by an intraperitoneal injection of sodium pentobarbital $(150 \mathrm{mg} / \mathrm{kg})$ after $12 \mathrm{~h}$ fasting. All procedures were approved by the Committee of Animal Use for Research and Teaching at The University of Hong Kong (approval no. CULATR No. 2787-12).
Quantitative nuclear magnetic resonance (qNMR). Measurements of the rats' body fat mass and composition by qNMR were calculated individually using a Brucker model mq10 NMR analyser (Brucker, Milton, Ontario, Canada). All data were recorded within a $1 \mathrm{~min}$ period.

Glucose tolerance test (GTT). In the 12th week, GTTs were performed in rats by injecting D-glucose (0.5 g/kg; Sigma-Aldrich; Merck KGaA) after $12 \mathrm{~h}$ fasting. The ACCU-CHEK blood glucose monitoring system (Roche Diagnostics) was used to examine the levels of glucose in the tail vein blood samples from each rat at $0,20,40,60,80,100$ and $120 \mathrm{~min}$ following glucose injection.

Insulin tolerance test (ITT). In the 12th week, rats were subjected to ITT after $12 \mathrm{~h}$ fasting and were injected with recombinant insulin (0.17 IU; cat\# 7544-MR-050, R\&D Systems, Inc.). Tail vein blood samples were collected for glucose level test at 0, 20, 40, 60, 80, 100 min following insulin injection using the ACCU-CHEK blood glucose monitoring system. Serum insulin levels were quantified by using High Sensitive Rat Insulin kit from Antibody and Immunoassay Services of The University of Hong Kong (cat\# 33270).

Histological studies. Liver and skeletal muscle tissues (hamstrings and gastrocnemius) were fixed for $72 \mathrm{~h}$ with $10 \%$ phosphate-buffered formalin and then embedded in paraffin blocks at ambient temperature. Following slicing of the tissues into 5- $\mu \mathrm{m}$ thick sections, they were stained with $0.1 \%$ haematoxylin and $0.1 \%$ eosin $(\mathrm{H} \& \mathrm{E})$ and $0.1 \%$ picro-Sirius Red (Polysciences Inc.) at ambient temperature as previously described (22). NAFLD activity scores were calculated to evaluate the hepatic histopathological injury based on the levels of steatosis, lobular inflammation, hepatocyte ballooning and fibrosis as previously described (23).

Immunohistochemistry (IHC). IHC staining was performed as described previously (24). Briefly, paraffin sections of skeletal muscle were processed and immunostained with glucose transporter 4 (GLUT4; Santa Cruz Biotechnology, Inc.) and inducible nitric oxide synthase (iNOS; BD Biosciences), using an $\mathrm{ABC}$ kit (Vector Laboratories Ltd.). The peroxidase was visualized using a DAB kit (Dako Denmark A/S; Agilent Technologies, Inc.). Negative control sections were incubated with secondary antibodies from Dako anti-mouse (cat\# K4000) or anti-rabbit (cat\# K4002). The presence of a brown chromogen indicated a positive staining result. Semi-quantitative assessment method was used in all IHC slides with $1+$ to $3+$ scoring $(10-30 \%$ staining $=1,31-60 \%=2$, $61-100 \%=3)$.

Oil red $O$ staining. Frozen muscle tissues were cut into 8-10 $\mu \mathrm{m}$ sections, fixed in $10 \%$ phosphate-buffered formalin for $72 \mathrm{~h}$ and stained with freshly prepared Oil Red $\mathrm{O}$ working solution for $15 \mathrm{~min}$ at ambient temperature. Nuclei were lightly stained with haematoxylin solution 5 dips as previously described (25).

Nuclear and cytoplasmic protein extraction. Nuclear and cytoplasmic proteins were extracted from skeletal muscle tissue using NE-PER Nuclear and Cytoplasmic Extraction 
Reagents kit (Pierce; Thermo Fisher Scientific, Inc.) with protease inhibitors aprotinin, leupeptin and pepstatin A (Sigma-Aldrich; Merck KGaA) according to the manufacturers' protocol.

Serum alanine aminotransferase (ALT) assay. Rat serum ALT levels were evaluated by using ALT (SGPT) reagent kits (Teco Diagnostics cat\# A524150) according to the manufacturer's protocol, to assess the overall hepatic injury caused by an HFD.

Malondialdehyde (MDA) assay. The levels of MDA were examined by Bioxytech LPO-586 ${ }^{\mathrm{TM}}$ kit (OXIS Health Products, Inc. cat\# 21012) according to the manufacturers' protocol. Skeletal muscle tissues were homogenised, followed by the determination of total protein concentration using protein assay kits (Bio-Rad Laboratories, Inc. cat\# 5000002) and an ELISA-based assay, with 1,1,3,3-tetraethoxypropane used to generate the standard curve.

RNA extraction and reverse transcription-quantitative polymerase chain reaction $(R T-q P C R)$. Total RNA from each liver sample was extracted using an illustra ${ }^{\mathrm{TM}}$ RNAspin mini kit (GE Healthcare Life Sciences), followed by RT processing using the SuperScript ${ }^{\mathrm{TM}}$ First-Strand Synthesis System (Invitrogen; Thermo Fisher Scientific, Inc.). The mRNA expression levels of lipogenic genes, fat-specific protein 27 (Fsp27), carbohydrate-responsive element-binding protein (ChREBP) and liver $\mathrm{X}$ receptor (LXR), were evaluated by Takara SYBR premix Taq qPCR system (Takara Bio, Inc) using a StepOnePlus ${ }^{\mathrm{TM}}$ Real-Time PCR instrument (Thermo Fisher Scientific, Inc.). GAPDH expression was used as a reference gene for normalisation. The thermocycle programs comprises initial denaturation $95^{\circ} \mathrm{C}$ for $30 \mathrm{sec}, 40$ cycles of denaturation at $95^{\circ} \mathrm{C}$ for $5 \mathrm{sec}$, annealing and elongation at $60^{\circ} \mathrm{C}$ for $30 \mathrm{sec}$, and final extension at $60^{\circ} \mathrm{C}$ for $30 \mathrm{sec}$. The efficiency and specificity of all primers were examined prior to the experiment. The expression levels of the target genes were calculated using the $2^{-\Delta \Delta \mathrm{Cq}}$ method (26) and then compared with the control group. The primer sequences for peroxisome proliferator-activated receptor $\alpha$ (PPAR $\alpha)$, Fsp27, ChREBP and LXR are listed in Table I.

Determination of serum free fatty acid (FFA) and triglyceride (TG). The levels of rat serum FFA were determined by an FFA fluorometric assay (cat\# 700310, Cayman Chemical Company) using a coupled enzymatic reaction, according to the manufacturers' protocol. Oleic acid was used for the preparation of the standard curve. Serum TG levels were determined by Serum Triglyceride Determination Kit according to manufacturers' protocol (cat\# TR0100, Sigma-Aldrich; Merck KGaA).

Western blot analysis. Western blot analyses were conducted as previously described (19). The dilution of all primary antibodies was 1:1,000. The following proteins were assessed: $\mathrm{NF}-\kappa \mathrm{B}$ (cat\# sc-372-G, Santa Cruz Biotechnology, Inc.); NF- $\kappa$ B inhibitor $\alpha$ (IкB $\alpha$; cat\# sc-1643, Santa Cruz Biotechnology, Inc.); interleukin (IL)-6 (cat\# sc-57315, Santa Cruz Biotechnology, Inc.); nuclear factor erythroid 2-related factor 2 (Nrf2; cat\# ab92946, Abcam); kelch-like ECH-associated protein 1 (Keap1; cat\# ab139729, Abcam); superoxide dismutase [Cu-Zn]
(SOD-1; cat\# sc-271014, Santa Cruz Biotechnology, Inc.); GAPDH (cat\# 2118, Cell Signaling Technology, Inc.); tripartite motif-containing family protein 72 (TRIM72; cat\# ab118651, Abcam); insulin receptor substrate 1 (IRS1, cat\# 3407, Cell Signaling Technology, Inc.); phosphorylated (Ser307) IRS1 (p-IRS1; cat\# 2381, Cell Signaling Technology, Inc.); PI3K (cat\# ab191606, Abcam); Akt (cat\# 4685, Cell Signaling Technology, Inc.); phosphorylated (Ser473) Akt (p-Akt; cat\# 4058, Cell Signaling Technology, Inc.); mTOR (cat\# 2983, Cell Signaling Technology, Inc.); phosphorylated (Ser307) mTOR (cat\# 2971, p-mTOR; Cell Signaling Technology, Inc.); and GLUT4 (cat\# sc-53566, Santa Cruz Biotechnology, Inc.). The dilution of anti-mouse IgG (H+L)-peroxidase (cat\# NA931) and anti-rabbit $\operatorname{IgG}(\mathrm{H}+\mathrm{L})$-peroxidase (cat\# NA934) antibodies was 1:2,000 (GE Healthcare). The optical density was read by Image J (Version 1.51j8; National Institutes of Health).

ELISA. The protein level of rat tumor necrosis factor- $\alpha$ (TNF- $\alpha$ ) was determined using a DuoSet ELISA kit (cat\# DY510, R\&D Systems, Inc.).

Statistical analysis. Data from each group $(n=6-9)$ are presented as mean \pm standard deviation. One-way analysis of variance (ANOVA) followed by a post-hoc Tukey's multiple comparisons test was performed to detect statistical differences between groups. For data from the body weight, GTT and ITT assays, a two-way ANOVA followed by a post-hoc Tukey's multiple comparisons test was performed to evaluate the statistical differences among groups at different time points. Kolmogorov-Smirnov test for normal distribution of data were performed. $\mathrm{P}<0.05$ was considered to indicate a statistically significant difference. All analyses were performed using IBM SPSS v.18 (SPSS, Inc.) software.

\section{Results}

A 4-week treadmill exercise regimen decreases body weight, fat mass and serum FFA levels, and rescues HFD-induced glucose and insulin intolerance. In the 9th week, rats began aerobic exercise, and in the following 4 weeks the body weight of rats in HFD + Exercise group decreased as compared with those in the HFD group (Fig. 1A). In the 12th week, body weight and fat mass were increased in the HFD diet only group, while the 4-week exercise treatment significantly decreased both body weight $(\mathrm{P}<0.001)$ and fat mass $(\mathrm{P}<0.01)$ in the HFD + exercise group (Fig. 1B and C). The elevated levels of serum FFA in HFD rats were also downregulated following exercise $(\mathrm{P}<0.01$; Fig. 1D). Increases in glucose and insulin intolerance were observed in the HFD rats, but these effects were significantly decreased following the exercise regimen (Fig. 1E and F). No difference in the ratio of liver weight and body weight in the rats in the present study was observed (data not shown).

Chronic exercise decreases hepatic lipid accumulation and lipogenesis, serum ALT and fat accumulation in muscle. There were severe liver injuries observed in the HFD rats, characterised by foci of inflammation and fat accumulation, as demonstrated by $\mathrm{H} \& \mathrm{E}$ staining of liver tissue, increases in serum ALT levels (65.54 IU/1 in HFD group) $(\mathrm{P}<0.001$; 
Table I. Primer sequences for reverse transcription-quantitative polymerase chain reaction.

\begin{tabular}{lll}
\hline Target gene & Direction & Sequence \\
\hline PPAR $\alpha$ & Forward & TACCACTATGGAGTCCACGCATG \\
& Reverse & TTGCAGCTTCGATCACACTTGTCG \\
ChREBP & Forward & GAAACCTGAGGCTGTTGTCTTG \\
& Reverse & TGTGGTATTCACGCATCA \\
Fsp27 & Forward & AGCTATCCCTTTCCCAGAAG \\
& Reverse & CCCTGTAGCAGTGCAGGTCA \\
LXR & Forward & CTGCAGGACAAAAAACTTCC \\
& Reverse & CCCTCCTCAGTCTGCTCCAC
\end{tabular}

PPAR $\alpha$, peroxisome proliferator-activated receptor $\alpha$; ChREBP, carbohydrate-responsive element-binding protein; Fsp27, fat-specific protein 27; LXR, liver X receptor.

A

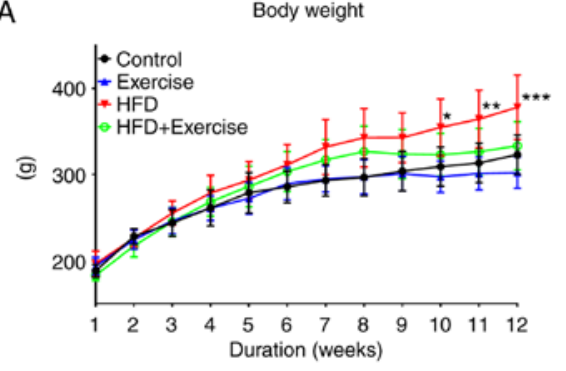

C

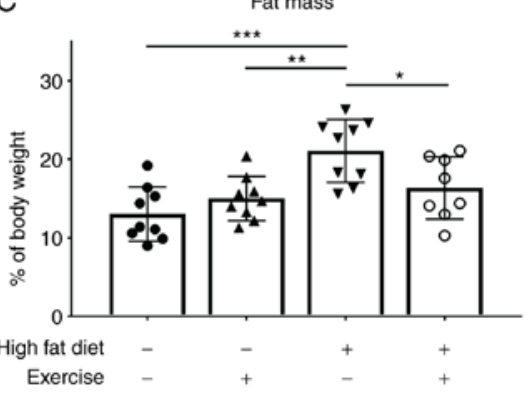

E

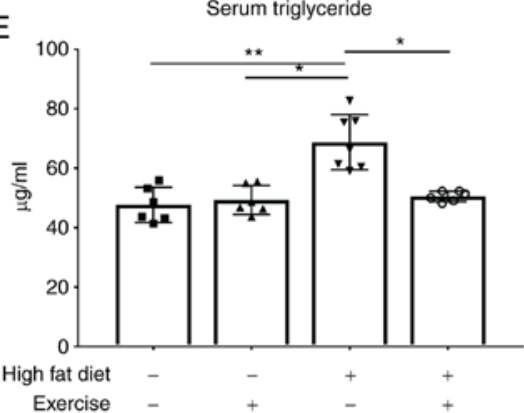

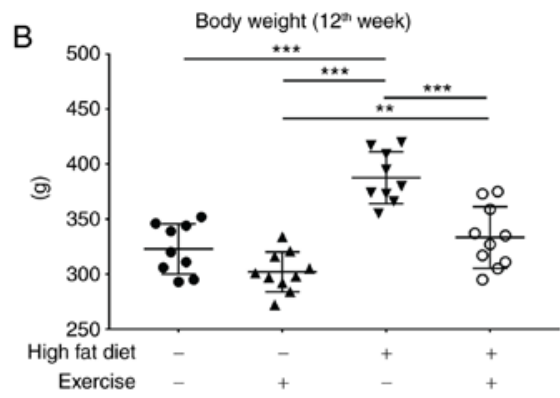

D
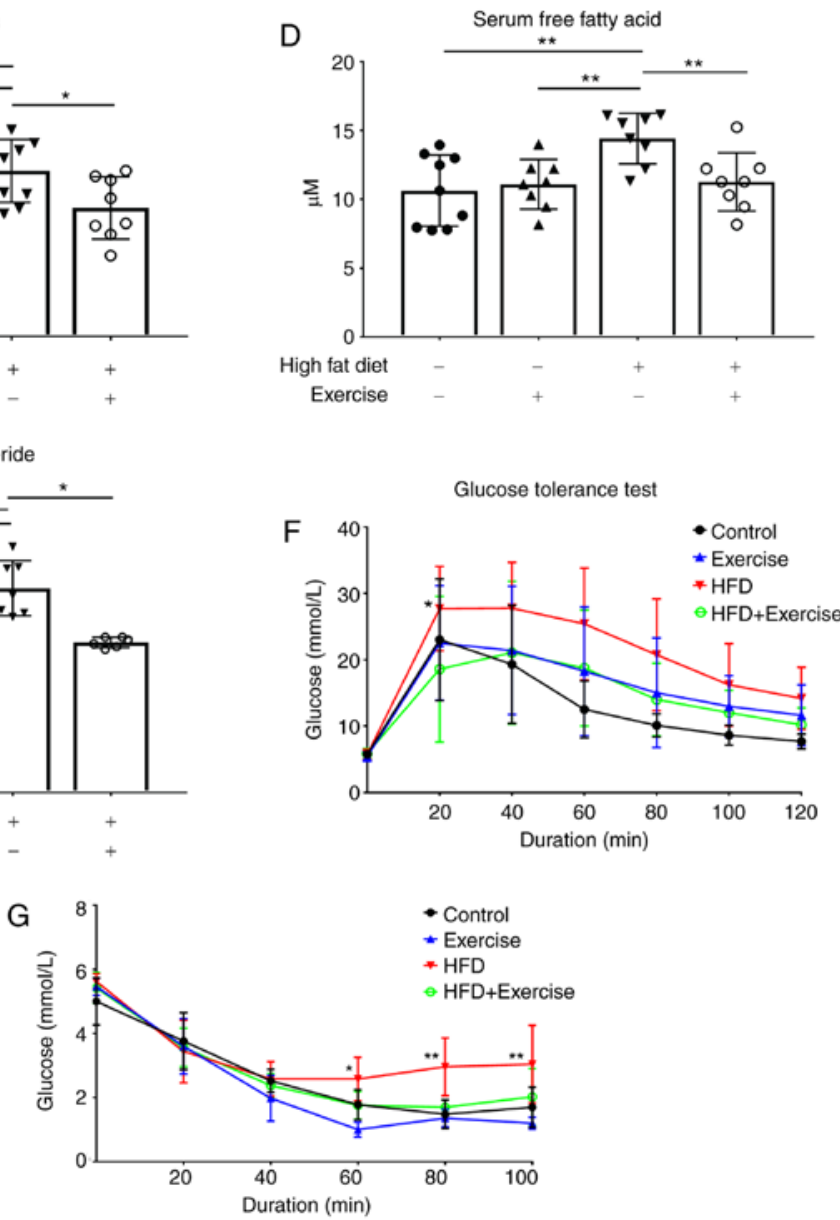

Figure 1. Effects of a 4-week aerobic exercise regimen on body weight for (A) 12 weeks and at (B) 12 weeks. (C) Fat mass, (D) serum free fatty acid levels and (E) serum triglyceride levels were measured in the 12th week. (F) Insulin tolerance tests and (G) glucose tolerance tests were performed at the indicated time points. Data are presented as mean \pm standard deviation. ${ }^{*} \mathrm{P}<0.05,{ }^{* * *} \mathrm{P}<0.01$ and ${ }^{* * * *} \mathrm{P}<0.001 \mathrm{~s}$. Control or as indicated. HFD, high-fat diet. 

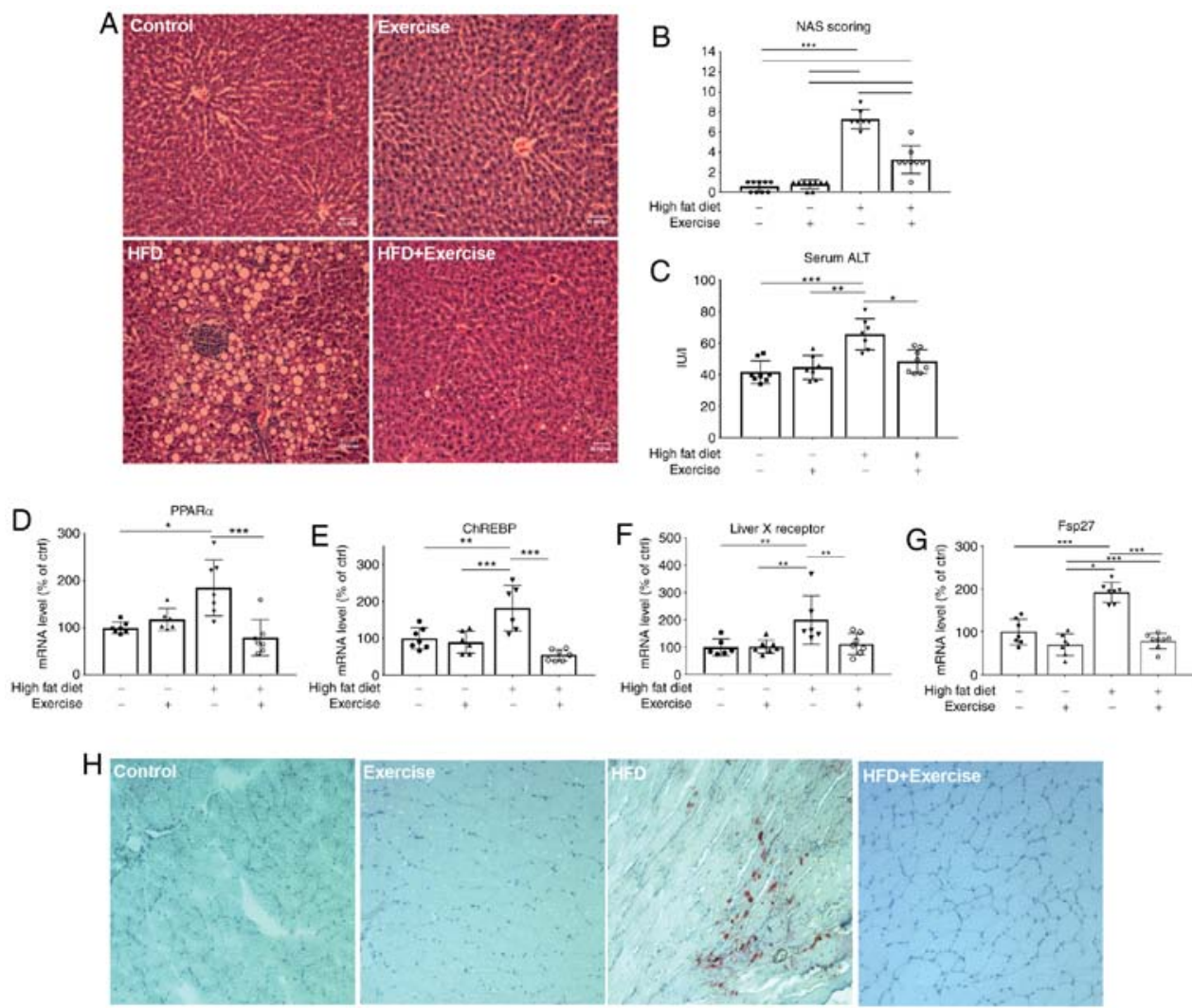

Figure 2. Effects of a 4-week aerobic exercise regimen. (A) Liver haematoxylin and eosin staining in the control, exercise, HFD and HFD + exercise groups (B) Corresponding NAS score. The levels of (C) liver injury marker serum ALT (IU/l), and mRNA expression levels of (D) PPAR , (E) ChREBP, (F) liver X receptor and (G) Fsp27 were measured. The mRNA expression data are presented as relative to the control group, which was arbitrarily assigned a value of 100. Data are presented as mean \pm standard deviation. ${ }^{*} \mathrm{P}<0.05,{ }^{* *} \mathrm{P}<0.01$ and ${ }^{* * *} \mathrm{P}<0.001$. (H) Muscle oil red $\mathrm{O}$ staining (magnification, $\left.\mathrm{x} 400\right)$. HFD, high-fat diet; NAS, non-alcoholic fatty liver disease activity scores; ALT, alanine aminotransferase; PPAR $\alpha$, peroxisome proliferator-activated receptor $\alpha$; ChREBP, carbohydrate-responsive element-binding protein; Fsp27, fat-specific protein 27.

Fig. 2A-C) and increases in fat accumulation in the skeletal muscle (Fig. 2G), which were remarkably reversed by exercise training. The elevated lipogenic markers PPAR $\alpha(\mathrm{P}<0.001)$, ChREBP $(\mathrm{P}<0.001)$, Fsp27 $(\mathrm{P}<0.01)$ and LXR $(\mathrm{P}<0.001)$ in HFD rats were suppressed after 4 weeks of exercise (Fig. 2D-F). No difference in muscle histology among the four groups was observed (data not shown).

Chronic aerobic exercise increases skeletal muscle insulin sensitivity partly through regulation of TRIM72 expression and PI3K/Akt/mTOR pathway with upregulation of GLUT4. The molecular mechanisms of the insulin signaling pathway in the skeletal muscle, which may contribute to whole-body insulin resistance, were then investigated. The skeletal muscles examined were hamstrings and gastrocnemius muscles, primarily made up of fast twitch muscle fibres and that no differences were observed between the muscles. TRIM72 is a key component in insulin resistance, and it may inhibit insulin signaling by targeting IRS and IRS1 for degradation in the skeletal muscle and heart (27-29). Skeletal muscle is a key tissue responsible for insulin-stimulated glucose consumption, so the present study measured the expression levels of TRIM72, IRS1, PI3K, Akt, mTOR and GLUT4 proteins. In the HFD rats, TRIM72 was significantly increased $(\mathrm{P}<0.01)$ while $\mathrm{p}$-IRS $(\mathrm{P}<0.05)$, PI3K $(\mathrm{P}<0.05)$, p-Akt $(\mathrm{P}<0.05)$, and GLUT4 $(\mathrm{P}<0.01)$ were decreased compared with rats in the control group, suggesting that there is an impairment of insulin signaling at the molecular level (Figs 3A-F). In addition, IHC staining also indicated decreased GLUT4 expression in the HFD group (Fig. 3H). Conversely, exercise alleviated the HFD-induced elevation of TRIM72, increased the expression levels of p-IRS1 $(\mathrm{P}<0.001)$ and GLUT4 $(\mathrm{P}<0.01)$, and restored the PI3K-Akt pathway, as evidenced by increases in $\mathrm{PI} 3 \mathrm{~K}(\mathrm{P}<0.05)$, p-Akt $(\mathrm{P}<0.01)$ and p-mTOR $(\mathrm{P}<0.01)$ expression levels (Fig. 3A-H), suggesting that exercise training may contribute to the improvement of glucose tolerance and systemic insulin sensitivity by stimulating glucose disposal and increasing insulin sensitivity in the skeletal muscle. However, no changes in IRS1 expression were observed (Fig. 3G).

\section{Exercise training ameliorates HFD-induced inflammation} via the $N F-\kappa B / I \kappa B \alpha$ pathway in skeletal muscle. $\mathrm{NF}-\kappa \mathrm{B}$ is a master regulator of the inflammation pathway; therefore, the protein expression levels of $\mathrm{NF}-\kappa \mathrm{B}$ in the nucleus, and the downstream inflammatory markers IL-6 and TNF- $\alpha$, were

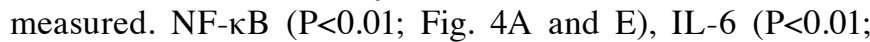
Fig. 4C and $\mathrm{E})$ and $\mathrm{TNF}-\alpha(\mathrm{P}<0.05$; Fig. 4D and $\mathrm{E})$ were increased in the HFD rats, while exercise training decreased the HFD-induced increase of these markers to the normal level. These results were additionally verified by corresponding levels of $\mathrm{I} \kappa \mathrm{B} \alpha$ protein expression in the cytoplasm (Fig. 4B and E). 

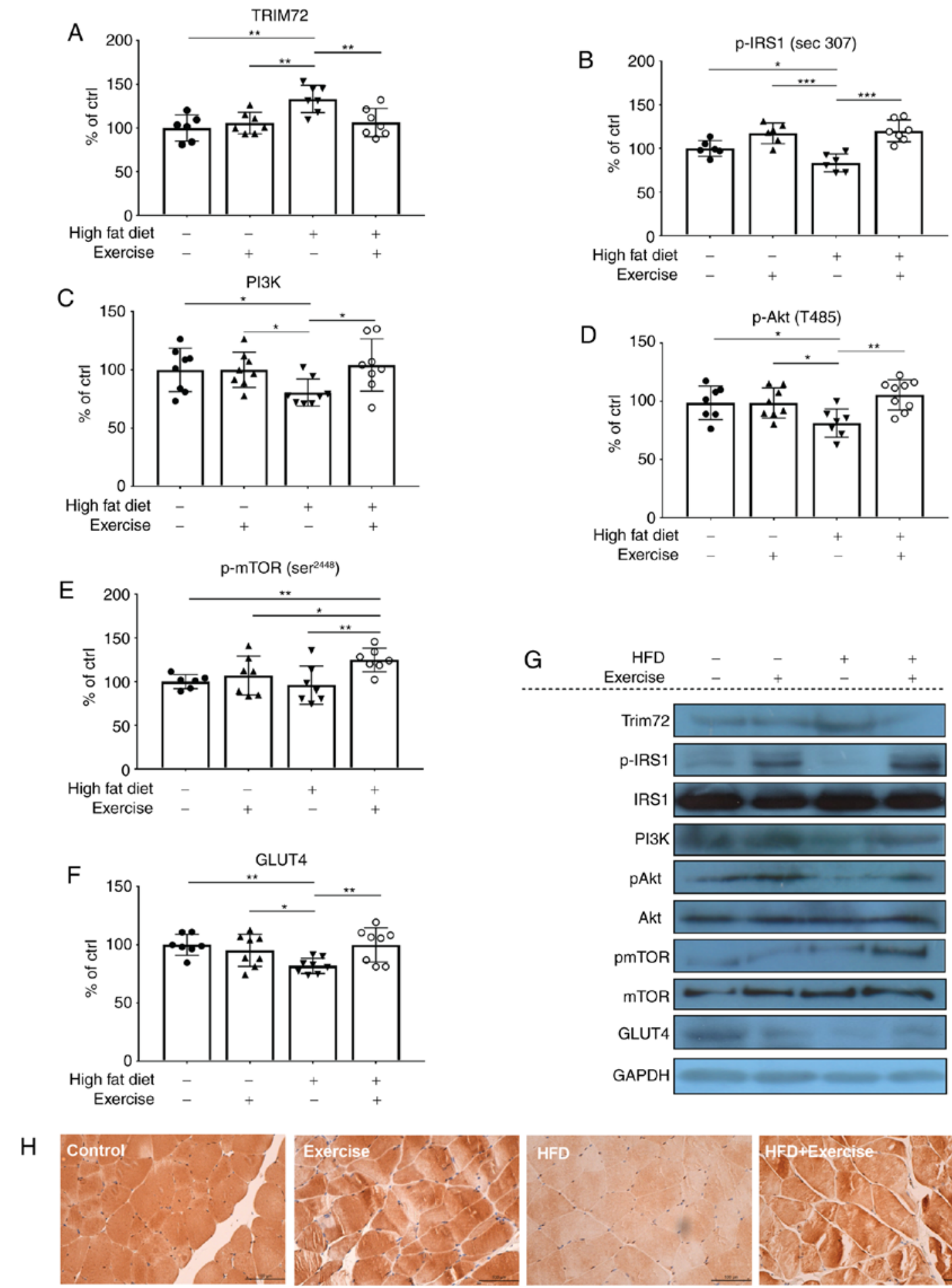

Figure 3. Protein expression levels of (A) TRIM72, (B) p-IRS1, (C) PI3K, (D) p-Akt, (E) p-mTOR and (F) GLUT4 were examined using western blot analysis. Data are presented as relative to the control group, which was arbitrarily assigned a value of 100 . Data are presented as mean \pm standard deviation. "P<0.05, ${ }^{* *} \mathrm{P}<0.01$ and ${ }^{* * *} \mathrm{P}<0.001$. (G) Representative western blot analysis gel using samples from in the skeletal muscles of the control, exercise, HFD and HFD + exercise groups. (H) Representative images of immunohistochemical staining of GLUT4 expression levels in the skeletal muscle (magnification, $\mathrm{x} 400$ ). HFD, high-fat diet; TRIM72, tripartite motif-containing family protein 72; IRS1, insulin receptor substrate 1; p-, phosphorylated; GLUT4, glucose transporter 4.

Chronic aerobic exercise activates Nrf2 and upregulates the antioxidant enzyme SOD-1 but fails to decrease oxidative and nitrosative stress in the skeletal muscle of NASH rats. To investigate whether exercise training was able to exert insulin-sensitizing effects partly via the decrease in oxidative stress, the antioxidant response and oxidative stress level were measured in the skeletal muscle. Nrf2 is a key transcription factor that regulates the antioxidant response. Notably, Nrf2 expression was significantly increased in the exercise rats with or without an HFD diet (Fig. 5A and D), suggesting that aerobic exercise acts on Nrf2, which may trigger the antioxidant response in the skeletal muscle. These results were additionally verified by examinations of Keapl protein expression in the cytoplasm (Fig. 5B and D). The level of SOD-1, 

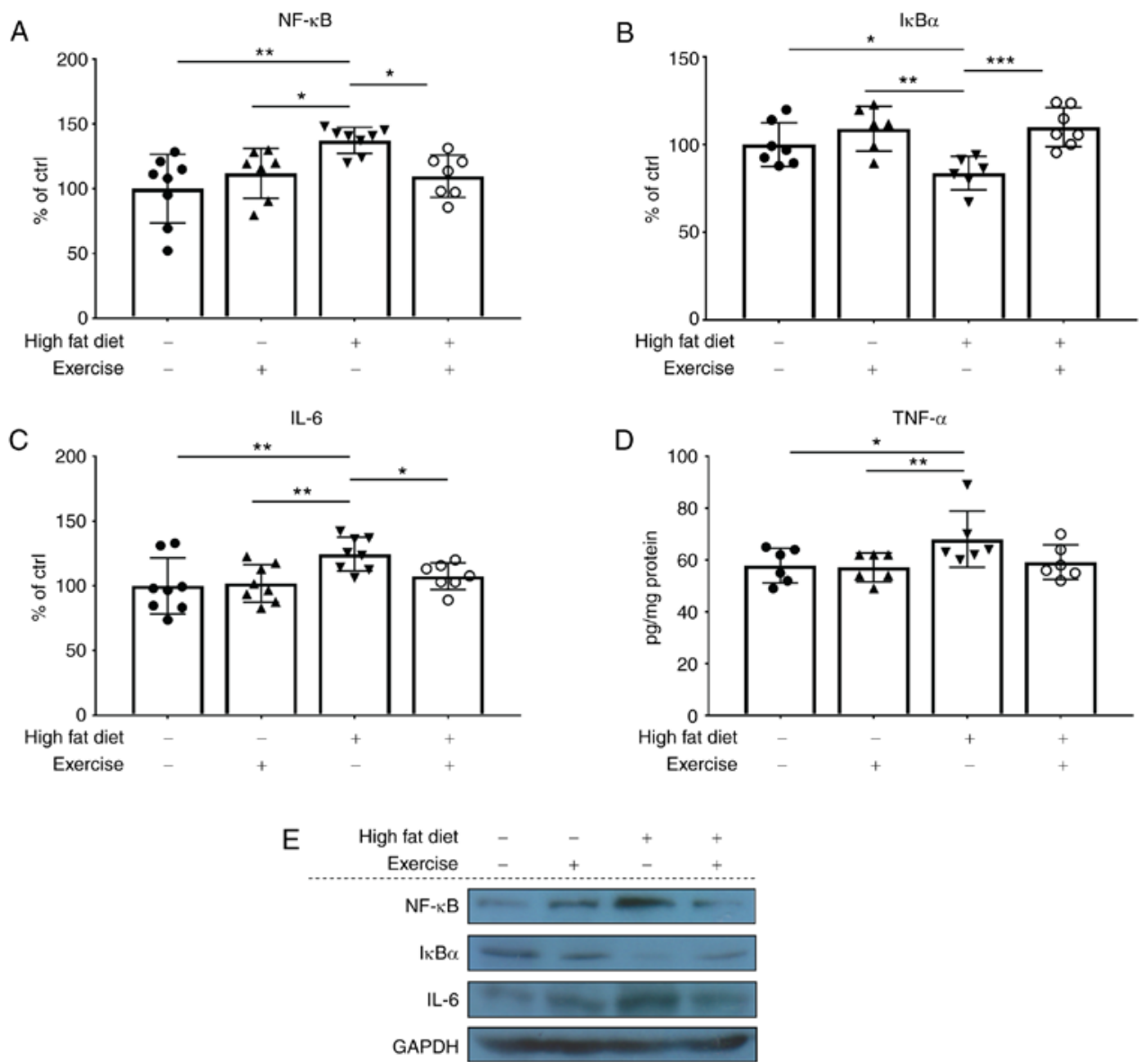

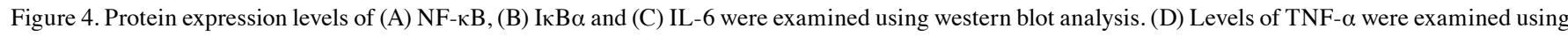
ELISA. (E) Representative western blot analysis gel using samples from in the skeletal muscles of the control, exercise, HFD and HFD + exercise groups. TNF- $\alpha$ data are presented as pg/mg protein. Western blot analysis data are presented as mean \pm standard deviation. ${ }^{*} \mathrm{P}<0.05,{ }^{* * *} \mathrm{P}<0.01$ and ${ }^{* * * *} \mathrm{P}<0.001$. The results are presented as relative to the control group, which was arbitrarily assigned a value of 100 . IкB $\alpha, \mathrm{NF}-\mathrm{kB}$ inhibitor $\alpha$; IL-6, interleukin-6; TNF- $\alpha$, tumor necrosis factor- $\alpha$; HFD, high-fat diet.

an antioxidant enzyme that is a downstream marker for Nrf-2, exhibited a similar trend to Nrf2 (Fig. 5C and D). However, exercise training failed to ameliorate the high levels of oxidative stress marker MDA (wHFD group=0.152 $\mu \mathrm{g} / \mathrm{mg}$; HFD + exercise group $=0.156 \mu \mathrm{g} / \mathrm{mg}$ ) and nitrosative stress marker iNOS in the skeletal muscle of HFD rats (Fig. 5E and F)

\section{Discussion}

TRIM72, also termed mitsugumin-53, was previously identified to participate in the cell membrane repair process, with specific expression in the skeletal muscle and heart compared with other tissues (27). Previously, Song et al (28) demonstrated that this protein inhibited insulin signaling by targeting IRS and IRS1 for degradation. To the best of our knowledge, the present study was the first to investigate the effects of chronic aerobic exercise on the expression level of TRIM72 in the skeletal muscle in a NASH model. The study performed by Qi et al (30) revealed that an 8-week swimming regimen markedly decreased the level of TRIM72 and increased the protein expression of $\mathrm{p}$-Akt, compared with the control. Swimming also improved the levels of total IRS1 whilst suppressing the HFD-induced phosphorylation of IRS1 on serine 307 (p-IRS1). In the present study, chronic aerobic exercise decreased the HFD-induced increase of TRIM72, maintained the levels of IRS1 phosphorylation, restored the PI3K/Akt/mTOR signaling pathway, and finally resulted in the increase in GLUT4 levels, which suggested an increase in glucose utilisation in the skeletal muscle and an improvement in systemic insulin sensitivity. However, the results observed for p-IRS1 and total IRS1 levels in the present study were inconsistent with previous data: Phosphorylation of IRS1 on serine residues has been suggested to mediate insulin resistance (31-34). Conversely, a previous study revealed that IRS1 Ser307 is in fact a positive regulatory site that promotes insulin sensitivity (35). It has been hypothesized that upregulation of Ser307 phosphorylation in mice with insulin resistance may be an adaptive response as opposed to a pathological change. Thus we proposed the inconsistent results observed may be as a result of the specific type of HFD used in the present study, which contains $30 \%$ fat from fish oil and has a pathogenic function in the muscle. As for total IRS1, although Song et al (28) concluded that TRIM72 targeted IR and IRS1 for degradation, a marked decrease in the HFD group was not observed, probably as the male mice in the study by Song et al (28) were fed with an HFD consisting of $60 \%$ calorie from fat for 35 weeks, while the animal model in the present study were all female rats fed 

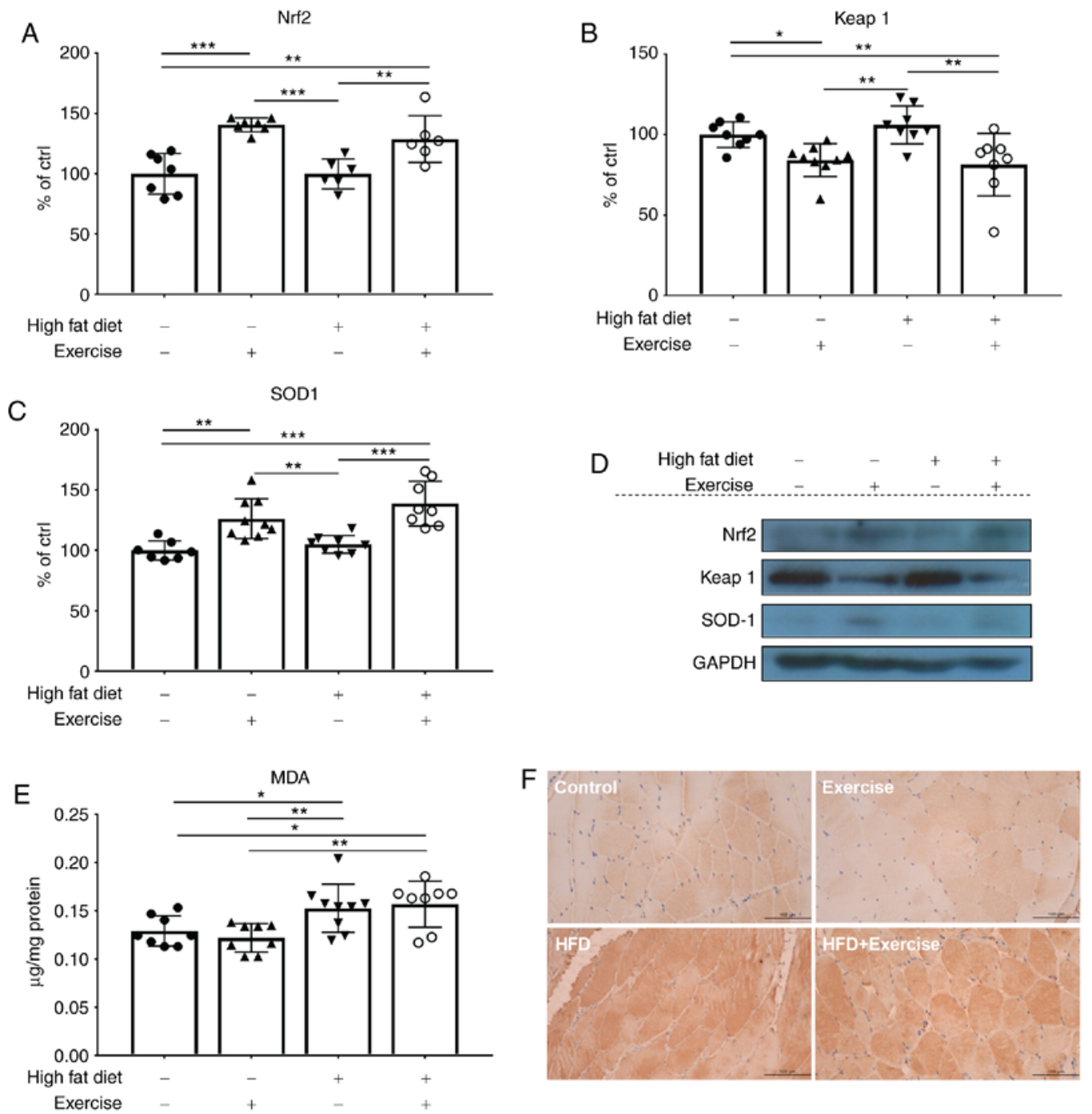

Figure 5. Protein expression levels of (A) Nrf2, (B) Keap1 and (C) SOD1 were examined using western blot analysis. (D) Representative western blot analysis gel using samples from in the skeletal muscles of the control, exercise, HFD and HFD + exercise groups. Western blot analysis data are presented as mean \pm standard deviation. ${ }^{*} \mathrm{P}<0.05,{ }^{* *} \mathrm{P}<0.01$ and ${ }^{* * * *} \mathrm{P}<0.001$. The results are presented as relative to the control group, which was arbitrarily assigned a value of 100. (E) The content of oxidative stress marker MDA is presented as $\mu \mathrm{g} / \mathrm{mg}$ protein. (F) Representative images of immunohistochemical staining of nitrosative stress marker inducible nitric oxide synthase (magnification, x400). Nrf2, nuclear factor erythroid 2-related factor 2; Keap1, kelch-like ECH-associated protein 1; SOD1, superoxide dismutase [Cu-Zn]; HFD, high-fat diet; MDA, malondialdehyde.

with an HFD that contained only $30 \%$ calories from fat (fish oil) for 12 weeks. It is possible that the inconsistencies between the data are a result of the differences between the sex, diet and duration of the animal models. However, there may be alternative explanations for these data, and further investigations are required.

The effects of exercise on the skeletal muscle are an integral part in the improvement of compromised systemic insulin resistance, which manifests in decreases in the levels of oxidative stress and inflammation. Nrf2 serves a pivotal role in the regulation of a series of genes encoding antioxidant systems under stress stimulus to adapt to ROS and xenobiotics $(36,37)$. A number of studies have demonstrated the beneficial effects of Nrf2 on various organs and tissues, including skeletal muscle, brain, kidney and liver (38-41). The data from the present study also demonstrated similar results, that a 4-week aerobic exercise regimen markedly activated the Nrf2 pathway and decreased Keap1 expression in the skeletal muscle tissues of the rats in the exercise and HFD + exercise groups. Therefore, consistent with previous studies, the results from the present study suggested that aerobic exercise may exert its beneficial effects on the improvement of insulin sensitivity partly through the improvement of the antioxidant response. However, chronic aerobic exercise did not decrease the level of lipid peroxidation product MDA in the skeletal muscle of HFD + exercise group, but instead increased MDA level by $20 \%$ compared with the control group. Consistently, exercise training failed to decrease the levels of nitrosative stress, as indicated by high level of iNOS. Numerous studies have demonstrated that exercise may decrease MDA levels in healthy individuals, however, in disease models such as hypercholesterolemia and diabetes, it has been suggested that exercise-induced changes in MDA were more pronounced $(42,43)$. Therefore, the possibility that although a 4-week aerobic exercise regimen increased the antioxidant response, it may not be sufficient to compensate the oxidative stress and nitrosative stress generated in the skeletal muscle, must not be dismissed. Nevertheless, the level of oxidative stress in the HFD + exercise group was only increased by $20 \%$ compared with the control group, with 
a significant increase in the level of antioxidant enzyme marker SOD1 as compared with the control group. In combination with the other beneficial effects of exercise observed in the HFD group, it may be concluded that the level of oxidative and nitrosative stress in the HFD + exercise group was inconsequential and not detrimental when compared with the HFD group.

In the animal model of the present study, rats fed with an HFD exhibited slight inflammation in the skeletal muscle. A number of studies have investigated the anti-inflammatory effects of chronic exercise in the skeletal muscle (44-48). It has been previously demonstrated that Nrf2 deletion may exaggerate inflammatory responses by inducing the expression of $\mathrm{NF}-\kappa \mathrm{B}, \mathrm{IL}-1 \beta$ and TNF- $\alpha$ in Nrf2 knockout mice fed with a methionine-choline deficient diet (49). Furthermore, in a recent study, Liu et al (50) identified that the hepatic insulin resistance was regulated by the $\mathrm{NF}-\kappa \mathrm{B}$ signaling pathway in $\mathrm{Nrf} 2$ knockout mice with NAFLD, indicating that Nrf2 deletion may lead to hepatic insulin resistance by activation of $N F-\kappa B$ pathway, Therefore, we hypothesized that the ameliorative effect of insulin resistance of chronic exercise may be mediated via the activation of $\mathrm{Nrf} 2$ and suppression of the $\mathrm{NF}-\kappa \mathrm{B}$ pathway, which are master regulators of the oxidative stress and inflammation pathway, respectively, and are closely associated.

In conclusion, to the best of our knowledge, the present study was the first to investigate the role of TRIM72 in alleviating insulin resistance during chronic aerobic exercise in a female NASH animal model fed with $30 \%$ of unsaturated fatty acids via fish oil. The results of the present study also supported the recently proposed hypothesis that skeletal muscle contributes to the improvement of systemic insulin resistance, therefore driving less postprandial glucose into hepatic lipogenesis, which may potentially alleviate fatty liver disease. Therefore, aerobic exercise is proposed as an important early lifestyle intervention to prevent fatty liver progression to steatohepatitis.

\section{Acknowledgements}

The authors would like to thank Ms. Carman Leung (School of Biomedical Sciences, The University of Hong Kong) for her technical help.

\section{Funding}

The present study was funded by the Seed Fund for Basic Research of University Research Committee (grant no. 20161159263).

\section{Availability of data and materials}

The datasets used and/or analysed during the current study are available from the corresponding author on reasonable request.

\section{Authors' contributions}

QY and GLT designed the experiments. EL assisted in the design of the study. QY and ZX conducted the experiments. QY and GLT analysed data, performed statistical tests and wrote the manuscript. All authors reviewed the final version of the manuscript.

\section{Ethics approval and consent to participate}

All procedures were approved by the Committee of Animal Use for Research and Teaching at The University of Hong Kong (approval no. CULATR No. 2787-12).

\section{Patient consent for publication}

Not applicable.

\section{Competing interests}

The authors declare that they have no competing interests.

\section{References}

1. Tilg $\mathrm{H}$ and Kaser A: Treatment strategies in nonalcoholic fatty liver disease. Nat Clin Pract Gastroenterol Hepatol 2: 148-155, 2005.

2. Krenkel O, Hundertmark J, Abdallah AT, Kohlhepp M, Puengel T, Roth T, Branco DPP, Mossanen JC, Luedde T, Trautwein C, et al: Myeloid cells in liver and bone marrow acquire a functionally distinct inflammatory phenotype during obesity-related steatohepatitis. Gut: May 10, 2019 (Epub ahead of print).

3. Jennison E, Patel J, Scorletti E and Byrne CD: Diagnosis and management of non-alcoholic fatty liver disease. Postgrad Med J 95: 314-322, 2019.

4. Duque-Guimarães DE and Ozanne SE: Nutritional programming of insulin resistance: Causes and consequences. Trends Endocrinol Metab 24: 525-535, 2013.

5. Flannery C, Dufour S, Rabøl R, Shulman GI and Petersen KF: Skeletal muscle insulin resistance promotes increased hepatic de novo lipogenesis, hyperlipidemia, and hepatic steatosis in the elderly. Diabetes 61: 2711-2717, 2012.

6. Jornayvaz FR, Samuel VT and Shulman GI: The role of muscle insulin resistance in the pathogenesis of atherogenic dyslipidemia and nonalcoholic fatty liver disease associated with the metabolic syndrome. Annu Rev Nutr 30: 273-290, 2010.

7. Kato K, Takeshita Y, Misu H, Zen Y, Kaneko S and Takamura T: Liver steatosis is associated with insulin resistance in skeletal muscle rather than in the liver in Japanese patients with non-alcoholic fatty liver disease. J Diabetes Investig 6: 158-163, 2015.

8. Rabøl R, Petersen KF, Dufour S, Flannery C and Shulman GI: Reversal of muscle insulin resistance with exercise reduces postprandial hepatic de novo lipogenesis in insulin resistant individuals. Proc Natl Acad Sci USA 108 8: 13705-13709, 2011.

9. Wellen KE and Hotamisligil GS: Inflammation, stress, and diabetes. J Clin Invest 115: 1111-1119, 2005.

10. de Luca and Olefsky JM: Inflammation and insulin resistance. FEBS Lett 582: 97-105, 2008.

11. Stump CS, Henriksen EJ, Wei Y and Sowers JR: The metabolic syndrome: Role of skeletal muscle metabolism. Ann Med 38: 389-402, 2006

12. Henriksen EJ: Exercise training and the antioxidant alpha-lipoic acid in the treatment of insulin resistance and type 2 diabetes. Free Radic Biol Med 40: 3-12, 2006.

13. Henriksen EJ, Diamond-Stanic MK and Marchionne EM: Oxidative stress and the etiology of insulin resistance and type 2 diabetes. Free Radic Biol Med 51: 993-999, 2011.

14. Johnson NA, Keating SE and George J: Exercise and the liver: Implications for therapy in fatty liver disorders. Semin Liver Dis 32: 65-79, 2012.

15. Keating SE, Hackett DA, George J and Johnson NA: Exercise and non-alcoholic fatty liver disease: A systematic review and meta-analysis. J Hepatol 57: 157-166, 2012.

16. Kistler KD, Brunt EM, Clark JM, Diehl AM, Sallis JF and Schwimmer JB; NASH CRN Research Group: Physical activity recommendations, exercise intensity, and histological severity of nonalcoholic fatty liver disease. Am J Gastroenterol 106: 460-468; quiz 469, 2011.

17. Lau JK, Zhang $\mathrm{X}$ and $\mathrm{Yu}$ J: Animal models of non-alcoholic fatty liver disease: Current perspectives and recent advances. J Pathol 241: 36-44, 2017. 
18. Takahashi Y, Soejima Y and Fukusato T: Animal models of nonalcoholic fatty liver disease/nonalcoholic steatohepatitis. World J Gastroenterol 18: 2300-2308, 2012.

19. Tipoe GL, Ho CT, Liong EC, Leung TM, Lau TY, Fung ML and Nanji AA: Voluntary oral feeding of rats not requiring a very high fat diet is a clinically relevant animal model of non-alcoholic fatty liver disease (NAFLD). Histol Histopathol 24: 1161-1169, 2009.

20. Liu Y, Li Q, Wang H, Zhao X, Li N, Zhang H, Chen G and Liu Z: Fish oil alleviates circadian bile composition dysregulation in male mice with NAFLD. J Nutr Biochem 69: 53-62, 2019.

21. Yamazaki T, Nakamori A, Sasaki E, Wada S and Ezaki O: Fish oil prevents sucrose-induced fatty liver but exacerbates high-safflower oil-induced fatty liver in ddy mice. Hepatology 46: 1779-1790, 2007.

22. Tipoe GL, Leung TM, Liong EC, Lau TY, Fung ML and Nanji AA: Epigallocatechin-3-gallate (EGCG) reduces liver inflammation, oxidative stress and fibrosis in carbon tetrachloride (CCl4)-induced liver injury in mice. Toxicology 273: 45-52, 2010.

23. Kleiner DE, Brunt EM, Van Natta, Behling C, Contos MJ, Cummings OW, Ferrell LD, Liu YC, Torbenson MS, Unalp-Arid a A, et al: Design and validation of a histological scoring system for nonalcoholic fatty liver disease. Hepatology 41: 1313-1321, 2005.

24. Liu Y, Tipoe GL and Fung ML: Melatonin attenuates intermittent hypoxia-induced lipid peroxidation and local inflammation in rat adrenal medulla. Int J Mol Sci 15: 18437-18452, 2014.

25. Chen B, Ma Y,Xue X, Wei J,Hu G and Lin Y: Tetramethylpyrazine reduces inflammation in the livers of mice fed a high fat diet. Mol Med Rep 19: 2561-2568, 2019.

26. Livak KJ and Schmittgen TD: Analysis of relative gene expression data using real-time quantitative PCR and the 2(-Delta Delta C(T)) method. Methods 25: 402-408, 2001.

27. Cao CM, Zhang Y, Weisleder N, Ferrante C, Wang X, Lv F, Zhang Y, Song R, Hwang M, Jin L, et al: MG53 constitutes a primary determinant of cardiac ischemic preconditioning. Circulation 121: 2565-2574, 2010.

28. Song R, Peng W, Zhang Y, Lv F, Wu HK, Guo J, Cao Y, Pi Y, Zhang X, Jin L, et al: Central role of E3 ubiquitin ligase MG53 in insulin resistance and metabolic disorders. Nature 494: 375-379, 2013.

29. Cai C, Masumiya H, Weisleder N, Matsuda N, Nishi M, Hwang M, Ko JK, Lin P, Thornton A, Zhao X, et al: MG53 nucleates assembly of cell membrane repair machinery. Nat Cell Biol 11: 56-64, 2009.

30. Qi J, Yang B, Ren C, Fu J and Zhang J: Swimming exercise alleviated insulin resistance by regulating tripartite motif family protein 72 expression and AKT signal pathway in sprague-dawley rats fed with high-fat diet. J Diabetes Res 2016: 1564386, 2016.

31. Hirosumi J, Tuncman G, Chang L, Görgün CZ, Uysal KT, Maeda K, Karin M and Hotamisligil GS: A central role for JNK in obesity and insulin resistance. Nature 420: 333-336, 2002.

32. Yi Z, Langlais P, De Filippis EA, Luo M, Flynn CR, Schroeder S, Weintraub ST, Mapes R and Mandarino LJ: Global assessment of regulation of phosphorylation of insulin receptor substrate-1 by insulin in vivo in human muscle. Diabetes 56: 1508-1516, 2007.

33. Yu C, Chen Y, Cline GW, Zhang D, Zong H, Wang Y, Bergeron R, Kim JK, Cushman SW, Cooney GJ, et al: Mechanism by which fatty acids inhibit insulin activation of insulin receptor substrate-1 (IRS-1)-associated phosphatidylinositol 3-kinase activity in muscle. J Biol Chem 277: 50230-50236, 2002.

34. Kruszynska YT, Worrall DS, Ofrecio J, Frias JP, Macaraeg G and Olefsky JM: Fatty acid-induced insulin resistance: Decreased muscle PI3K activation but unchanged Akt phosphorylation. J Clin Endocrinol Metab 87: 226-234, 2002.

35. Copps KD, Hancer NJ, Opare-Ado L, Qiu W, Walsh C and White MF: Irs1 serine 307 promotes insulin sensitivity in mice. Cell Metab 11: 84-92, 2010.
36. Tebay LE, Robertson H, Durant ST, Vitale SR, Penning TM, Dinkova-Kostova AT and Hayes JD: Mechanisms of activation of the transcription factor Nrf2 by redox stressors, nutrient cues, and energy status and the pathways through which it attenuates degenerative disease. Free Radic Biol Med 88: 108-146, 2015.

37. Chanas SA, Jiang Q, McMahon M, McWalter GK, McLellan LI, Elcombe CR, Henderson CJ, Wolf CR, Moffat GJ, Itoh K, et al: Loss of the Nrf2 transcription factor causes a marked reduction in constitutive and inducible expression of the glutathione S-transferase Gsta1, Gsta2, Gstm1, Gstm2, Gstm3 and Gstm4 genes in the livers of male and female mice. Biochem J 365: 405-416, 2002.

38. Asghar M, George L and Lokhandwala MF: Exercise decreases oxidative stress and inflammation and restores renal dopamine D1 receptor function in old rats. Am J Physiol Renal Physiol 293: F914-F919, 2007.

39. Done AJ and Traustadóttir T: Nrf2 mediates redox adaptations to exercise. Redox Biol 10: 191-199, 2016.

40. Muthusamy VR, Kannan S, Sadhaasivam K, Gounder SS, Davidson CJ, Boeheme C,Hoidal JR, Wang L and Rajasekaran NS: Acute exercise stress activates Nrf2/ARE signaling and promotes antioxidant mechanisms in the myocardium. Free Radic Biol Med 52: 366-376, 2012.

41. Zhao X, Bian Y, Sun Y, Li L, Wang L, Zhao C, Shen Y, Song Q, $\mathrm{Qu} Y$, Niu S, et al: Effects of moderate exercise over different phases on age-related physiological dysfunction in testes of SAMP8 mice. Exp Gerontol 48: 869-880, 2013.

42. Sen C, Packer L and Hänninen O: Handbook of oxidants and antioxidants in exercise. Elsevier Science 2000.

43. Kim JS and Yi HK: Intermittent bout exercise training down-regulates age-associated inflammation in skeletal muscles. Exp Gerontol 72: 261-268, 2015.

44. Cechella JL, Leite MR, Dobrachinski F, da Rocha JT, Carvalho NR, Duarte MM, Soares FA, Bresciani G, Royes LF and Zeni G: Moderate swimming exercise and caffeine supplementation reduce the levels of inflammatory cytokines without causing oxidative stress in tissues of middle-aged rats. Amino Acids 46: 1187-1195, 2014

45. Isanejad A, Saraf ZH, Mahdavi M, Gharakhanlou R, Shamsi MM and Paulsen G: The effect of endurance training and downhill running on the expression of IL-1ß, IL-6, and TNF- $\alpha$ and HSP72 in rat skeletal muscle. Cytokine 73: 302-308, 2015

46. Jeong JH, Park HG, Lee YR and Lee WL: Moderate exercise training is more effective than resveratrol supplementation for ameliorating lipid metabolic complication in skeletal muscle of high fat diet-induced obese mice. J Exerc Nutrition Biochem 19: 131-137, 2015.

47. Tantiwong P, Shanmugasundaram K, Monroy A, Ghosh S, Li M, DeFronzo RA, Cersosimo E, Sriwijitkamol A, Mohan S and Musi N: NF-B activity in muscle from obese and type 2 diabetic subjects under basal and exercise-stimulated conditions. Am J Physiol Endocrinol Metab 299: E794-E801, 2010.

48. Knudsen JG, Joensen E, Bertholdt L, Jessen H, van Hauen L, Hidalgo $J$ and Pilegaard H: Skeletal muscle IL-6 and regulation of liver metabolism during high-fat diet and exercise training. Physiol Rep 4: e12788, 2016.

49. Chowdhry S, Nazmy MH, Meakin PJ, Dinkova-Kostova AT, Walsh SV, Tsujita T, Dillon JF, Ashford ML and Hayes JD: Loss of Nrf2 markedly exacerbates nonalcoholic steatohepatitis. Free Radic Biol Med 48: 357-371, 2010.

50. Liu Z, Dou W, Ni Z, Wen Q, Zhang R, Qin M, Wang X, Tang H, Cao Y, Wang J and Zhao S: Deletion of Nrf2 leads to hepatic insulin resistance via the activation of NF-B in mice fed a high-fat diet. Mol Med Rep 14: 1323-1331, 2016.

This work is licensed under a Creative Commons Attribution-NonCommercial-NoDerivatives 4.0 International (CC BY-NC-ND 4.0) License. 